\title{
PREVALÊNCIA DE CONSTIPAÇÃO INTESTINAL EM UNIVERSITÁRIAS DO CURSO DE FISIOTERAPIA
}

\author{
INTESTINAL CONSTIPATION PREVALENCE IN PHYSICAL \\ THERAPY UNIVERSITY STUDENTS
}

\author{
Guilherme Tavares de Arruda', Melissa Medeiros Braz²
}

\section{RESUMO}

Esta pesquisa teve como objetivo investigar a prevalência de constipação intestinal entre as acadêmicas do curso de Fisioterapia. Foi realizada pesquisa descritiva com 144 acadêmicas do curso de Fisioterapia da Universidade Federal de Santa Maria. Foi aplicado o questionário de Roma III para determinar a prevalência de constipação intestinal entre as acadêmicas. Os dados foram tratados por meio da estatística descritiva. Dentre as pesquisadas, 88 (61,12\%) apresentavam constipação intestinal. Dos sintomas mais relevantes, verificou-se que $93,18 \%$ das acadêmicas relataram sensação de evacuação incompleta, $77,27 \%$ sensação de obstrução ou bloqueio anorretal, 44,31\% forçar para evacuar, $42,04 \%$ apresentavam fezes duras ou fragmentadas, $22,72 \%$ utilizavam de manobras manuais para facilitar a evacuação e $35,22 \%$ relataram frequência de menos de três evacuações por semana. Houve alta prevalência de constipação intestinal no grupo analisado, o que torna necessária a intervenção da equipe de saúde para contribuir no tratamento desta disfunção, estimulando o autocuidado e hábitos saudáveis.

Descritores: Constipação Intestinal; Mulheres; Estudantes de Ciências da Saúde; Saúde da Mulher.
${ }^{1}$ Acadêmico de Fisioterapia pela Universidade Federal de Santa Maria (UFSM), Santa Maria, RS, Brasil.

2 Doutora em Engenharia de Produção e Sistemas/Ergonomia pela Universidade Federal de Santa Catarina (UFSC), Florianópolis, SC, Brasil.

\begin{abstract}
This research aimed to investigate the prevalence of constipation among the academic of Physiotherapy course. Descriptive study was conducted with 144 academic of Federal University of Santa Maria. The Rome III questionnaire was used to determine the prevalence of constipation among academic. Data were treated using descriptive statistics. Among the surveyed, $88(61.12 \%)$ had constipation. From relevant symptoms, it was found that $93.18 \%$ of academic reported feeling of incomplete evacuation, feeling of $77.27 \%$ anorectal obstruction or blockage, forcing to evacuate $44.31 \%, 42.04 \%$ had hard or lumpy stools, $22.72 \%$ used for manual maneuvers to facilitate the evacuation and $35.22 \%$ reported frequency of less than three bowel movements per week. There was a high prevalence of chronic constipation in the analyzed group, the intervention of the health team makes it necessary to contribute in treating this dysfunction, stimulating self-care and healthy habits.
\end{abstract}

Descriptors: Constipation; Women; Students Health Occupations; Women's Health. 


\section{Introdução}

Constipação intestinal $(\mathrm{Cl})$ pode ser definida como uma síndrome baseada em sintomas como defecação insatisfatória, com dificuldade na passagem das fezes ou evacuações infrequentes, fezes duras ou sensação de evacuação incompleta, que pode ocorrer isolada ou secundariamente a uma doença subjacente ${ }^{1}$.

Estudo realizado no Brasil com estudantes universitários aponta uma prevalência de $40 \%$ associados a fatores de risco como histórico familiar e uso de laxativos ${ }^{2}$. A prevalência de $\mathrm{Cl}$ entre as estudantes universitárias brasileiras foi reportada entre $14 \% 2$ e $24,4 \%{ }^{3}$.

$\mathrm{A} \mathrm{Cl}$ é uma condição patológica que possui repercussões no assoalho pélvico feminino. $\mathrm{O}$ esforço evacuatório crônico leva ao estiramento da musculatura perineal e das paredes do reto, ocasionando incontinências urinária (IU) e fecal (IF) 4 . O prolapso genital seria outra patologia associada à constipação, pois o esforço ao evacuar distenderia o ramo motor do nervo pudendo levando a este agravo ${ }^{5}$.

A existência de poucos estudos sobre o tema aliada ao conhecimento dos dados apresentados atentou-nos para a interferência do tema sobre a saúde da mulher. 0 número de outras doenças que podem ser prevenidas com acompanhamento adequado para com a mulher constipada também motivou a realização da pesquisa.

Esta pesquisa teve como objetivo investigar a prevalência de constipação intestinal entre as acadêmicas do curso de Fisioterapia.

\section{Metodologia}

A pesquisa foi do tipo descritiva. Foi aprovada pelo Comitê de Ética em Pesquisa da UFSM sob CAAE 07853412.2.0000.5346.

A população consistiu de estudantes universitárias do sexo feminino de 18 a 35 anos. A prevalência de $\mathrm{Cl}$ foi investigada com a totalidade das acadêmicas do curso de graduação em Fisioterapia da Universidade Federal de Santa Maria (UFSM), RS que aceitaram participar da pesquisa.

Como critérios de exclusão, constavam: gestantes, mulheres que possuam distúrbios neurológicos centrais ou periféricos, alterações de sensibilidade cutânea, patologias gastrointestinais previamente diagnosticadas, hipertermia, cicatrizes abdominais, cirurgias abdominais prévias e que estivessem em uso de laxativos.

Foram utilizados como instrumentos de coleta de dados:

Questionário de Roma III para constipação ${ }^{7}$, utilizado na avaliação da $\mathrm{Cl}$ e foi escolhido por representar uma forma padronizada internacionalmente para o diagnóstico da constipação e também por ser uma forma atualizada do questionário de Roma II, utilizado anteriormente em pesquisas sobre o assunto. Este questionário possui 17 perguntas referentes ao trânsito intestinal e sintomatologia relacionada.

Os critérios para avaliação de constipação funcional através do Questionário de Roma III foram aqueles utilizados internacionalmente:

\section{As respostas devem incluir dois ou mais dos critérios abaixo:}

a) forçar em pelo menos $25 \%$ das evacuações (questão $11>1$ )

b) fezes duras ou fragmentadas em pelo menos $25 \%$ das evacuações (questão 10>1)

c) sensação de evacuação incompleta em pelo menos $25 \%$ de todas as evacuações (questão 12>0)

d) sensação de obstrução anorretal ou bloqueio em pelo menos $25 \%$ das evacuações (questão 13>0)

e) manobras manuais para facilitar a evacuação em pelo menos $25 \%$ das evacuações (questão 14>0)

f) menos de 3 evacuações por semana (questão $9>1$ )

2. Fezes líquidas raramente estariam presentes sem o uso de laxantes. Fezes líquidas deviam ocorrer nunca ou raramente (questão 17=0)

3. Critérios insuficientes para IBS (síndrome do intestino irritável). 
Ficha de avaliação adaptada de Henscher ${ }^{6}$, a qual possui 11 questões abertas sobre distúrbios de evacuação na mulher, além de dados antropométricos (massa corporal e altura auto-referidos).

O questionário de Roma III foi distribuído pelos pesquisadores e respondido pelas acadêmicas do curso de Fisioterapia em um horário previamente agendado, nas salas de aula do curso de Fisioterapia.

Para o tratamento dos dados foi realizada a estatística descritiva. Os dados foram tabulados e tratados no software Excel versão 2007.

\section{Resultados e Discussão}

Foram pesquisadas 144 universitárias com idades variando entre 17 e 34 anos $(20,44 \pm 2,68)$. Quanto ao índice de massa corporal (IMC) das participantes, 9 participantes não forneceram seus dados antropométricos. Das 135 respondentes, o IMC variou entre 14,27 e $29,41 \mathrm{~kg} / \mathrm{m}^{2}(21,44 \pm 2,45)$.

Entre as 144 pesquisadas, $88(61,12 \%)$ apresentavam constipação intestinal. Os sintomas mais prevalentes são apresentados na tabela 1. A sensação de evacuação incompleta foi encontrada em 93,18\% da amostra e a sensação de obstrução anorretal ou bloqueio em 77,27\%.

Tabela 1 - Sintomas da constipação intestinal, de acordo com o questionário de Roma III, em pelo menos $25 \%$ das evacuações.

\begin{tabular}{c|c}
\hline Sintomas & $\begin{array}{c}\text { N (\%) } \\
\mathbf{N}=\mathbf{8 8}\end{array}$ \\
\hline Forçar para evacuar & $39(44,31 \%)$ \\
\hline Fezes duras ou fragmentadas & $37(42,04 \%)$ \\
\hline $\begin{array}{c}\text { Sensação de evacuação incompleta } \\
\text { Sensação de obstrução anorretal ou } \\
\text { bloqueio }\end{array}$ & $82(93,18 \%)$ \\
\hline $\begin{array}{c}\text { Manobras manuais para facilitar a } \\
\text { evacuação }\end{array}$ & $20(22,72 \%)$ \\
\hline $\begin{array}{c}\text { Necessidade de uso de laxantes para } \\
\text { evacuar }\end{array}$ & $11(12,64 \%)$ \\
\hline $\begin{array}{c}\text { Sensação de flatulência na região } \\
\text { abdominal }\end{array}$ & $56(67,46 \%)$ \\
\hline Menos de 3 evacuações por semana & $31(35,22 \%)$ \\
\hline
\end{tabular}

No presente estudo, a prevalência de $\mathrm{Cl}$ foi de $61,12 \%$, achado inferior ao de um estudo realizado em uma instituição federal de ensino superior do Norte de Minas $^{2}$, no qual 142 alunos foram selecionados ao acaso com faixa etária de 18 a 24 anos. Constatou-se que todas as mulheres do estudo (100\%) apresentavam constipação, sendo que $90 \%$ delas ingeriam menos de 1,5 litro de líquidos por dia. Outro aspecto a se observar foi o alto índice de dificuldade de evacuar fora de casa que as constipadas possuíam (85\%). Sugere-se que a alta prevalência de $\mathrm{Cl}$ está relacionada a maus hábitos alimentares, principalmente à baixa ingesta de água por dia, o que torna o bolo fecal mais petrificado $\mathrm{e}$ de difícil evacuação; e ao aumento do tempo fora de casa, que faz com que o indivíduo espere para chegar ao lar para evacuar com mais comodidade.

Um estudo realizado em uma universidade da China com 5000 estudantes $^{8}$, de ambos os sexos e com idade entre 18 e 23 anos, mostrou que 429 estudantes (237 do sexo feminino) apresentaram $\mathrm{Cl}$. Houve prevalência de $\mathrm{Cl}$ entre as mulheres e os sintomas mais relatados foram menos de 3 evacuações por semana $(98,8 \%)$, evacuação incompleta $(97,6 \%)$, esforço para evacuar $(92,3 \%)$, fezes duras ou irregulares $(88,8 \%)$ e obstrução anorretal $(88,8 \%)$. Comparados ao atual estudo, a sensação de evacuação incompleta, o esforço para evacuar e a sensação de obstrução anorretal ou bloqueio também se mostraram importantes. 
Há evidente ligação do surgimento de IU e IF com as pressões intra-abdominais causadas pelo esforço para evacuar e a sensação de obstrução anorretal em mulheres após os 35 anos $^{9,10}$. Sabe-se que a IU e IF diminuem a qualidade de vida dessas mulheres, levando à diminuição do contato social e da autoestima por causa da perda involuntária de urina e/ou fezes.

Estudo transversal realizado com 360 estudantes de medicina de uma instituição do noroeste paulista ${ }^{11}$, utilizando as respostas de um questionário relativo à $\mathrm{Cl}$, observou que dos 150 estudantes que responderam ao questionário, houve prevalência de $\mathrm{Cl}$ de $35 \%$. Destes, $55 \%$ são jovens adultos do sexo feminino com média de $\mathrm{IMC}$ de $21,89 \mathrm{~kg} / \mathrm{m}^{2}$. Concluiu-se que além de haver maior prevalência de $\mathrm{Cl}$ nas mulheres, também houve uso mais frequente de laxativos entre as mulheres constipadas (46\%). Apesar da média do IMC encontrada nesse estudo ${ }^{11}$ e na atual investigação estar na faixa de peso normal em mulheres constipadas, destaca-se que o aumento do IMC é um dos fatores que ocasiona $\mathrm{Cl}$, o que não é evidenciado em nosso estudo.

O uso de laxativos $(33,34 \%)$ também foi relatado com maior destaque ${ }^{12}$, em estudo do tipo transversal realizado com estudantes de Nutrição de um centro universitário no leste de Minas Gerais. Dos 40 estudantes incluídos no estudo, $22,5 \%$ (todos do sexo feminino) eram constipados. Assim como no presente estudo, o qual foi menos frequente entre os constipados a necessidade do uso de laxativos para evacuar (12,64\%), no estudo realizado com 2946 entrevistados na população da cidade de Pelotas/RS, houve também um baixo uso de laxativos $(22,4 \%)$ entre os indivíduos que referiram utilizar alguma ajuda para evacuar ${ }^{13}$.

Foi realizada pesquisa sobre hábitos alimentares e intestinais em estudantes universitários da área da saúde da Universidade Municipal de São Caetano do Sul (IMES) ${ }^{14}$. Foram entrevistados 181 alunos com idade média de 20 anos. Destes, $67,9 \%$ eram mulheres e $17 \%$ do total apresentavam $\mathrm{Cl}$, sendo prevalente nas mulheres (64\%). 0 esvaziamento incompleto foi a maior queixa relatada $(41,9 \%)$, achado semelhante ao nosso estudo. Outras queixas relatadas foram a presença de sangue nas fezes $(6,5 \%)$, o esforço em excesso para evacuar $(9,6 \%)$ e a presença de dor $(13 \%)$. Observouse também que o consumo de fibra era insuficiente entre os constipados. Porém, não foi encontrada nenhuma relação entre estado nutricional e hábito intestinal.

Os sistemas urinário, genital e intestinal estão intrinsecamente relacionados na função do trato urinário e na sustentação anatômica. Devido a isso, há ligação entre $\mathrm{Cl}$ e o surgimento de IU e IF em idades avançadas. Sabe-se que esses transtornos têm grande efeito sobre a qualidade de vida do paciente: reduzem as atividades físicas e sociais, além de causar isolamento e comprometer sua autoestima. Portanto, é preciso formular estratégias para a melhora do trânsito intestinal destes jovens e assim reduzir o risco de IU e IF no futuro. A avaliação propedêutica e terapêutica da $\mathrm{Cl}$, juntamente com a equipe de saúde objetivando a melhora nos resultados do tratamento, se fazem necessárias.

\section{Considerações Finais}

Diante dos resultados, foi alta a prevalência de $\mathrm{Cl}$, se comparada a outros estudos. Os sintomas mais relatados foram a sensação de evacuação incompleta, a sensação de obstrução anorretal ou bloqueio, a sensação de flatulência na região abdominal e o esforço para evacuar. Ressalta-se a importância da atuação do profissional de saúde em orientar hábitos alimentares saudáveis e incentivar a prática de exercícios físicos a fim de melhorar a qualidade de vida desses indivíduos.

\section{Referências}

1. Bharucha A, Pemberton J, Locke G. American Gastroenterological Association technical review on constipation. Gastroenterology. 2013;144(1):218-38.

2. Cota RP, Miranda LS. Associação entre constipação intestinal e estilo de vida em estudantes universitários. RevBrasNutrClin. 2006; 21(4):296-301. Disponível em: <http://www.sbnpe.com.br> Acesso em: 23 abril 2008.

3. Magalhães ACP, et al. Prevalência e fatores de risco para constipação intestinal em estudantes universitários. Nutrpauta. 2002;(56):13-17.

4. Brading AP, Turner WH. The unstable bladder: towards a common mechanism. Br J Urol. 1994;73:3-8.

5. Dvorkin LS, Knowles CH, Scott SM, Williams NS, Lunniss PJ. Rectal intussusception: characterization of symptomatology. Dis Colon Rectum 2005; 48: 824-31.

6. Henscher U. Fisioterapia em Ginecologia. São Paulo: Santos; 2007. 
7. Rome foundation: The Functional Gastrointestinal Disorders. Constipation module. Questionários de diversas disfunções gastrointestinais. Disponível em: <http://www.romecriteria.org> Acesso em: abril 2008.

8. Dong Y-Y, Chen F-X, Yu Y-B, Du C, Qi Q-Q, Liu H, et al. A school-based study with Rome III criteria on the prevalence of functional gastrointestinal disorders in Chinese college and university students. PLoS One [Internet]. 2013 Jan [cited 2014 Oct 19];8(1):e54183. Disponível em: http://www.pubmedcentral.nih.gov/articlerender. fcgi?artid=3548818\&tool=pmcentrez\&rendertype=abstract

9. Bharucha A, Wald A. Transtornos anorretais. Arq Gastroenterol [Internet]. 2012 [cited 2014 Nov 2];49:51-60. Disponível em: http://www.scielo.br/scielo.php?pid=S0004-28032012000500009\&script=sci_arttext\&tlng=es

10. Korelo R, Kosiba C, Grecco L, Matos R. Influência do fortalecimento abdominal na função perineal, associado ou não à orientação de contração do assoalho pélvico, em nulíparas. Fisioter Mov [Internet]. 2011 [cited 2014 Nov 2];24(1):7585. Disponível em: http://www.scielo.br/pdf/fm/v24n1/v24n1a09.pdf

11. Trisóglio C, Marchi CMG, Torres U dos S, Netinho JG. Prevalência de Constipação Intestinal entre Estudantes de Medicina de uma Instituição no Noroeste Paulista. Rev Bras Coloproctol. 2010;30(2):203-9.

12. Braz CR de S, Lessa NMV. Fatores de risco e prevalência de constipação intestinal em graduandos do curso de nutrição de um centro universitário no leste de Minas Gerais. Nutr gerais. Ipatinga; 2011;5(8):740-54.

13. Collete VL, Araújo CL, Madruga SW. Prevalência e fatores associados à constipação intestinal: um estudo de base populacional em Pelotas, Rio Grande do Sul, Brasil, 2007. Cad Saúde Pública. Rio de Janeiro; 2010;26(7):1391-402.

14. Martinoff T, Aquino R de $C$ de. Avaliação de constipação intestinal e sua relação com hábito alimentar e estilo de vida de universitários. Rev Bras Ciências da Saúde. 2008;3(15):47-51.

\section{Guilherme Tavares de Arruda}

Endereço para correspondência - Rua: General Osório, n³30, Bairro: Centro, CEP: 97060-270, Santa Maria, RS, Brasil.

E-mail: gui_tavares007@hotmail.com

Lattes: http://lattes.cnpq.br/3473188802626741

Melissa Medeiros Braz - melissabraz@hotmail.com

Enviado em 26 de janeiro de 2016. Aceito em 15 de setembro de 2016. 